\title{
Craniospinal irradiation as a salvage treatment for metastatic relapsed DIPG
}

\author{
Marta Perez-Somarriba ${ }^{1}$, Vicente Santa-María López ${ }^{2}$, Ofelia Cruz Martinez ${ }^{1}$, Jordi \\ Muchart $^{1}$, Cinzia Lavarino ${ }^{1}$, Soraya Mico $^{3}$, and Andres Morales La Madrid ${ }^{1}$ \\ ${ }^{1}$ Hospital Sant Joan de Deu \\ ${ }^{2}$ Hospital Sant Joan de Déu \\ ${ }^{3}$ Hospital Vall d'Hebron
}

August 23, 2020

\begin{abstract}
Diffuse intrinsic pontine glioma (DIPG) is the most frequent brainstem tumor in children. All therapeutic efforts are palliative, being focal irradiation the only therapy capable of reducing temporarily tumor volume. The vast majority of patients succumb to their disease during the first 2 years after diagnosis usually due to local progression. At first relapse, focal re-irradiation has shown to provide clinical and radiologic benefits. There are no reports of craniospinal irradiation (CSI) as a rescue regimen when progression is local and disseminated. We report a patient treated with CSI at metastatic progression showing clinical and radiological response with good tolerance.
\end{abstract}

\section{Introduction:}

Diffuse intrinsic pontine glioma (DIPG) is an aggressive lethal brainstem tumor that occurs predominantly in the pediatric population, accounting for nearly $80 \%$ of tumors in this location ${ }^{1-3}$. Currently, focal radiation therapy (RT) is the only intervention that temporarily halts tumor progression with clinical and radiologic responses in the majority of cases. ${ }^{4}$ However, most tumors progress after a few months of upfront irradiation. ${ }^{2,3}$ Although critical molecular alterations and biologic pathways have been identified in the last decade, ${ }^{5}$ no effective drugs have been introduced into the clinic. At first progression, focal reirradiation (reRT) seems to offer clinical and radiological benefit to patients, particularly to those with initial and maintained response to irradiation. ${ }^{6}{ }^{2,7}$ However, no clear recommendations exist in the context of metastatic progression. Anecdotally, CSI has been used upfront in the treatment of a few metastatic DIPG patients alone or in combination with other treatments. ${ }^{8-10}$ To our knowledge, there are no reports of its use at first metastatic progression.

Case report

A previously healthy 10-year old boy presented with 2 weeks of ataxia and left facial palsy. Brain MRI showed an expansive T1 hypointense/T2 hyperintense non-enhancing diffuse infiltrating mass located within the pons, images compatible with a DIPG. No leptomeningeal dissemination was identified at diagnosis (Fig. $1 \mathrm{~A})$.

An open biopsy was performed as part of our institutional precision oncology program for tumors with unfavorable prognosis without complications. The histology and molecular studies confirmed a K27M mutated diffuse midline glioma (H3.3), TP53 wild type. No therapeutic targets were identified.

He initiated treatment with normofractionated focal irradiation up to a total dose of 54Gy. Treatment was well tolerated with marked clinical improvement, being able to perform daily life activities and discontinuing 
steroids by the end of irradiation. No adjuvant therapy was administered.

Ten months after the end of radiation, a surveillance MRI showed subtle local progression associated with leptomeningeal dissemination with multiple new intraventricular nodules identified in several MRI sequences. The patient was asymptomatic, so closely follow-up without treatment was recommended.

Five months later, patient developed slight worsening of ataxia and left facial palsy. The MRI showed progressive disease both locally and metastatically. (Fig. 1C) (Fig. 2A)

He initiated palliative CSI receiving 21,6 Gy with good tolerance and clinical improvement. The MRI after reRT showed a good response of all lesions. (Fig. 1D)(Fig. 2B).

Importantly, quality of life remained good and patient could go back to normal day activities.

Four months after the end of the reRT, neurologic symptoms (left hemiparesis and ataxia) worsened with subsequent MRI showing disease progression.

Patient died of disease at home followed closely by our pediatric palliative care team 24 months after diagnosis, 12 months after first radiologic metastatic progression, and 6 months after craniospinal reRT .

Discussion

Macroscopic leptomeningeal dissemination at DIPG progression has been reported in up to $30 \%$ of cases. $10-13$

However, the real number is probably underestimated because a high proportion of patients at the end of life are not imaged and/or do not undergo an autopsy. Furthermore, when histologically studied, microscopic disease dissemination has been found in a greater proportion. Caretti et al examined autopsy material from 16 DIPG patients and observed subventricular dissemination in $63 \%$ of the patients. Consequently, the real overall incidence of at distance dissemination is not well established, but likely higher than reported.5,12,14

ReRT, although palliative, has shown to be a good salvage in a number of contexts (i.e. relapsed HGG, ependymoma) given its reasonable tolerance and clinical/radiologic benefit. ${ }^{1}{ }^{7}$ Fontanilla et alreported 6 patients treated with focal reRT (20Gy) at recurrence for the first time in $2012 .^{2}$ Since then, several groups have reported similar positive experiences. ${ }^{3,151}$. Recently, Lu et al reported a meta-analysis on this topic reinforcing the role of palliative focal reRT specifically for DIPG. ${ }^{16}$ In patients with DIPG, focal progression is usually the rule and is responsible for most of the neurologic symptoms and ultimate clinical deterioration, reinforcing the use of focal reRT. Our case is singular because the patient presented metastatic asymptomatic progression. However, and as in other DIPG cases without dissemination, with further tumor growth he developed brainstem symptomatology. Given the presence of other tumoral lesions and the good initial disease response to upfront irradiation, we opted to administer CSI, being our goal to control both disease compartments (local and leptomeningeal). Fortunately our approach was very well tolerated, proved clinical and radiological response, extending his survival for 6 months. Of note, quality of life was remarkably good with no need for admissions and with good functionality until his last days.

Based on this experience, we conclude that until new and effective therapies are identified, CSI could be a safe and good palliative reRT strategy for patients with progressed disseminated DIPG.

Conflicts of interest: The authors declare no conflicts of interest

\section{References:}

1. Amsbaugh MJ, Mahajan A, Thall PF, et al: A Phase 1/2 Trial of Reirradiation for Diffuse Intrinsic Pontine Glioma. Int J Radiat Oncol Biol Phys 104:144-148, 2019

2. Fontanilla HP, Pinnix CC, Ketonen LM, et al: Palliative reirradiation for progressive diffuse intrinsic pontine glioma. Am J Clin Oncol 35:51-7, 2012 
3. Freese C, Takiar V, Fouladi M, et al: Radiation and subsequent reirradiation outcomes in the treatment of diffuse intrinsic pontine glioma and a systematic review of the reirradiation literature. Pract Radiat Oncol $7: 86-92,2017$

4. Gallitto M, Lazarev S, Wasserman I, et al: Role of Radiation Therapy in the Management of Diffuse Intrinsic Pontine Glioma: A Systematic Review. Adv Radiat Oncol 4:520-531, 2019

5. Kluiver TA, Alieva M, van Vuurden DG, et al: Invaders Exposed: Understanding and Targeting Tumor Cell Invasion in Diffuse Intrinsic Pontine Glioma. Front Oncol 10:92, 2020

6. Janssens GO, Gandola L, Bolle S, et al: Survival benefit for patients with diffuse intrinsic pontine glioma (DIPG) undergoing re-irradiation at first progression: A matched-cohort analysis on behalf of the SIOP-E-HGG/DIPG working group. Eur J Cancer 73:38-47, 2017

7. Morales La Madrid A, Santa-Maria V, Cruz Martinez O, et al: Second re-irradiation for DIPG progression, re-considering "old strategies" with new approaches. Childs Nerv Syst 33:849-852, 2017

8. Muller K, Schlamann A, Guckenberger M, et al: Craniospinal irradiation with concurrent temozolomide for primary metastatic pediatric high-grade or diffuse intrinsic pontine gliomas. A first report from the GPOH-HIT-HGG Study Group. Strahlenther Onkol 190:377-81, 2014

9. Muller K, Schlamann A, Seidel C, et al: Craniospinal irradiation with concurrent temozolomide and nimotuzumab in a child with primary metastatic diffuse intrinsic pontine glioma. A compassionate use treatment. Strahlenther Onkol 189:693-6, 2013

10. Wagner S, Benesch M, Berthold F, et al: Secondary dissemination in children with high-grade malignant gliomas and diffuse intrinsic pontine gliomas. Br J Cancer 95:991-7, 2006

11. Tinkle CL, Orr BA, Lucas JT, Jr., et al: Rapid and fulminant leptomeningeal spread following radiotherapy in diffuse intrinsic pontine glioma. Pediatr Blood Cancer 64, 2017

12. Gururangan S, McLaughlin CA, Brashears J, et al: Incidence and patterns of neuraxis metastases in children with diffuse pontine glioma. J Neurooncol 77:207-12, 2006

13. Vanan MI, Eisenstat DD: DIPG in Children - What Can We Learn from the Past? Front Oncol 5:237, 2015

14. Caretti V, Bugiani M, Freret M, et al: Subventricular spread of diffuse intrinsic pontine glioma. Acta Neuropathol 128:605-7, 2014

15. Lassaletta A, Strother D, Laperriere N, et al: Reirradiation in patients with diffuse intrinsic pontine gliomas: The Canadian experience. Pediatr Blood Cancer 65:e26988, 2018

16. Lu VM, Welby JP, Mahajan A, et al: Reirradiation for diffuse intrinsic pontine glioma: a systematic review and meta-analysis. Childs Nerv Syst 35:739-746, 2019

17. Cooney TM, Cohen KJ, Guimaraes CV, et al: Response assessment in diffuse intrinsic pontine glioma: recommendations from the Response Assessment in Pediatric Neuro-Oncology (RAPNO) working group. The Lancet Oncology 21:e330-e336, 2020

Figure legends:

Figure 1. T2-weighted MRI, Axial sections, sequences at the level of the pons.

1. Diagnostic MRI shows high T2 signal and enlargement of the pons encasing the basilar artery and left lateral extension towards the cerebellar peduncle and left cerebellar hemisphere consistent with DIPG.

2. 3 month-follow up MRI after surgical biopsy and radiation therapy shows significant reduction of the pontine tumefaction and cerebellar peduncle extension compatible with partial response.

3. 15 months after first irradiation, there is new enlargement of the pons with high signal consistent with progressive disease. 
4. MRI after reirradiation. There is a new radiological improvement with reduction of the pons size and less high T2 signal compatible with partial response. ${ }^{17}$

Figure 2. Sagittal FLAIR + post-gadolinium at relapse (A) and after re-irradiation (B).

Both the primary brainstem lesion and the metastasis (anterior recesses of the III ventricle, in relation with the corpus callosum and mid-line frontal lobes) show volume

reduction and improvement of signal abnormality.
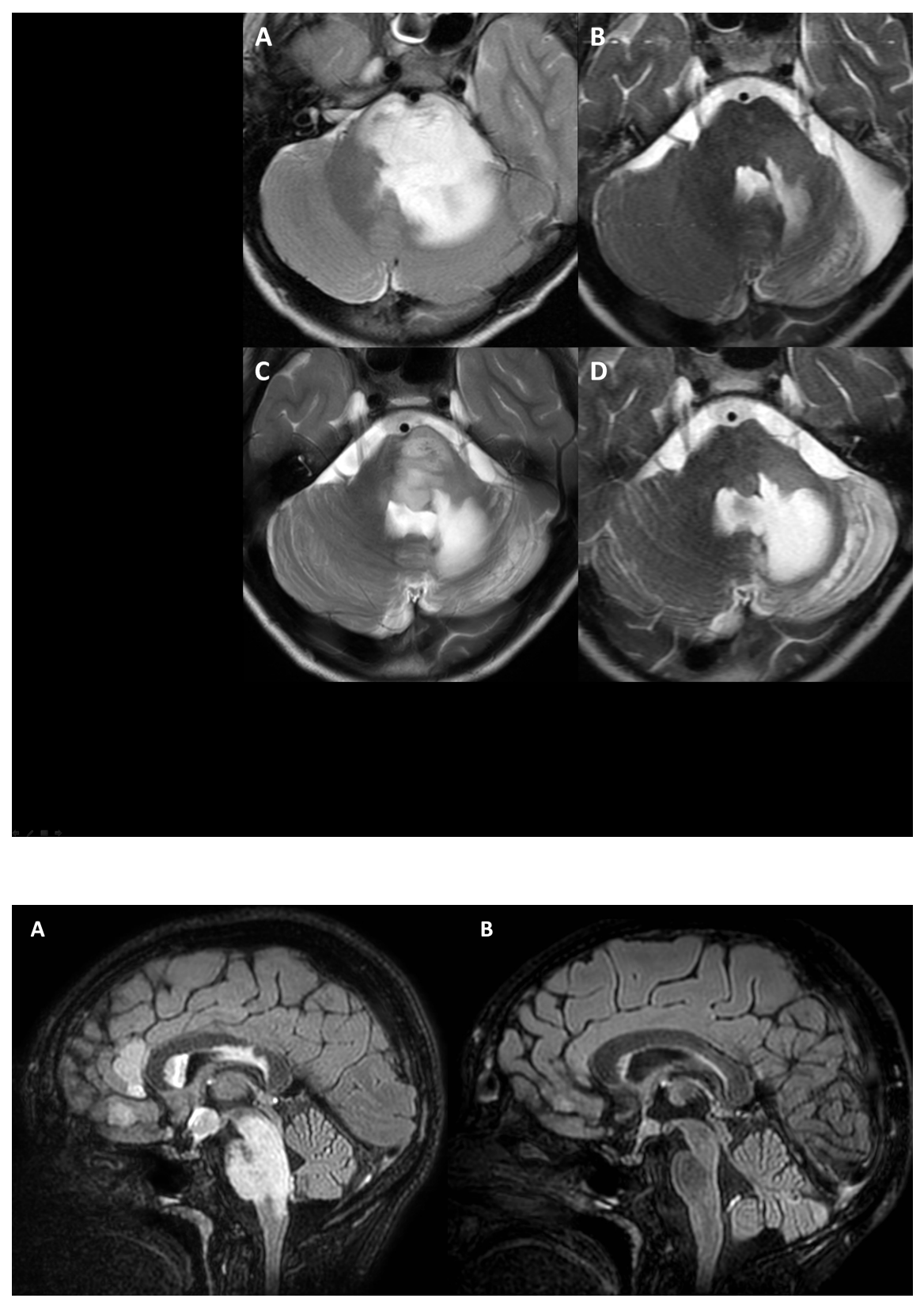